\title{
Efficacy of the Novel Degludec/Aspart Insulin Co-formulation in Children and Adolescents with Type 1 Diabetes: A Real-life Experience with One Year of IDegAsp Therapy in Poorly Controlled and Non-compliant Patients
}

\author{
(D) Tarık Kırkgöz, (D) Mehmet Eltan, (D) Sare Betül Kaygusuz, (D) Zehra Yavaş Abalı, (D) Didem Helvacıoğlu, (D) Tuba Seven Menevşe, \\ (D) Büşra Gürpınar Tosun, (D) Tülay Güran, (D) Abdullah Bereket, (D) Serap Turan
}

Marmara University Faculty of Medicine, Department of Pediatric Endocrinology, İstanbul, Turkey

\section{What is already known on this topic?}

Achieving optimal metabolic control can be extremely challenging in some children and adolescents with type 1 diabetes (T1DM). Adherence to multiple insulin injections is poor in a subgroup of these children, leading to frequent hospitalization because of diabetic ketoacidosis (DKA). Degludec/aspart co-formulation (70\% IDeg + 30\% IAsp - IDegAsp) can be beneficial in challenging cases with poor glycemic control and acute complications of diabetes by providing the longer-duration of basal insulin with simplified basal-bolus treatment in 3 injections instead of 4-5 injections.

\section{What this study adds?}

The real-life experience demonstrates that IDegAsp is non-inferior to classic basal-bolus regimen regarding to glycemic control in children with T1DM. Simplified basal-bolus regimen with IDegAsp could be an alternative in patients with frequent hypoglycemia and DKA attacks, who have poor compliance with 4-5 injections per day.

\footnotetext{
Abstract

Objective: To evaluate the efficacy of degludec/aspart (IDegAsp) insulin co-formulation in children and adolescents with poorly controlled type 1 diabetes (T1DM).

Methods: Patients with poorly controlled T1DM on basal-bolus insulin regimes and having compliance problems related to insulin injections were switched to IDegAsp and were included. Data on hemoglobin A1c (HbA1c) levels, hypoglycemic episodes, frequency of diabetic ketoacidosis (DKA) and insulin doses were recorded at baseline and after one year of IDegAsp treatment.

Results: Fifty patients (22 girls; $44 \%$ ) were started on IDegAsp. The mean \pm standard deviation (range) age and duration of diabetes were $12.9 \pm 3.4(4-18)$ and $5.2 \pm 3.1$ (1.0-13.7) years, respectively. At the end of one year, 38 patients were still on IDegAsp, whereas 12 patients had opted to resume their original treatments. In those who continued on IDegAsp, HbA1c levels did not change, but the number of self-reported mild-moderate hypoglycemic episodes decreased significantly $(p<0.05)$. In the year before switching to IDegAsp, 11 DKA attacks in 9 patients were observed, whereas this decreased to 4 DKA attacks in 4 patients after one year of IDegAsp therapy $(p=0.06)$.

Conclusion: IDegAsp regimen may improve clinical management in poorly controlled basal-bolus insulin regimen T1DM patients who have frequent hypoglycemia and DKA attacks, as well as in those with poor compliance with multiple injections. Although a simplified basal-bolus IDegAsp regimen is an attractive option for patients with T1DM, some may not adapt to this treatment due to the fixed IAsp dose of IDegAsp.
}

Keywords: Type 1 diabetes mellitus, hypoglycemia, diabetic ketoacidosis, co-formulation, insulin degludec, insulin aspart, IDegAsp

Address for Correspondence: Serap Demircioğlu Turan MD, Marmara University Faculty of Medicine, Department of Pediatric Endocrinology, İstanbul, Turkey Phone: + 902166254545 E-mail: serap.turan@marmara.edu.tr ORCID: orcid.org/0000-0002-5172-5402

${ }^{\circ}$ Copyright 2022 by Turkish Pediatric Endocrinology and Diabetes Society

The Journal of Clinical Research in Pediatric Endocrinology published by Galenos Publishing House.
Conflict of interest: None declared Received: 04.05.2021 Accepted: 03.08.2021 


\section{Introduction}

Currently, basal-bolus insulin regimes are the most commonly used treatment modality in children and adolescents with type 1 diabetes mellitus (T1DM) worldwide. Insulin pump therapy, although permitting a more physiological insulin delivery, is not available to all patients due to high cost that hinders patient access to treatment $(1,2,3)$.

Optimal glycemic control is considered the key factor in reducing the risk of long term microvascular and macrovascular complications in diabetes patients (1), while only $14-30 \%$ of the patients are reported to achieve target hemoglobin A1c (HbA1c) levels (2). Furthermore, achievement of glycemic control is more challenging in an adolescent population due to poor patient compliance with anti-diabetic treatment and insulin injections in particular $(2,3)$. Accordingly, these patients are more prone to acute complications of T1DM such as recurrent episodes of diabetic ketoacidosis (DKA) and hypoglycemia $(2,3)$. Given that a standard basal-bolus insulin regime requires 4-5 injections per day, increasing the likelihood of omitting insulin injections, longer acting basal insulins seem to offer an alternative treatment approach with a potential to enable better glycemic control and reduced risk of recurrent DKA in children and adolescents with T1DM and poor treatment compliance. New long-acting insulin analogue degludec (IDeg), developed by removal of threonine at B30 of human insulin and adding a glutamic acid spacer to a 16-carbon diacid at B29, has a mean half-life of 25 hours, while the half-life of the longest acting insulin (glargine) on the market is 12 hours (4). IDeg is considered to offer stable coverage of basal insulin needs due to its flatter and more consistent pharmacodynamic profile with a duration of action exceeding 42 hours and fourfold less within-subject variability compared to insulin glargine $(5,6)$. Furthermore, IDeg can be mixed with rapid acting insulin analogueinsulin aspart (IAsp) without affecting the pharmacokinetics of either molecule, while other long acting insulin analogues cannot be mixed or co-formulated $(5,6)$.

Insulin degludec/insulin aspart (IDegAsp) combining $70 \%$ IDeg and $30 \%$ IAsp, is a soluble combination of two individual insulin analogues in one product, designed to provide mealtime glycemic control through the IAsp component and basal glucose-lowering effect through the IDeg component. IDegAsp could provide flat and stable basal insulin coverage (provided by IDeg at steady-state conditions) and bolus mealtime insulin control with reduced injection burden compared to standard basal and bolus therapy (7). In addition, IDegAsp has been approved for use in T1DM patients over two years of age by European
Medicine Agency and one year of age and older by the U.S. Food and Drug Administration DA $(8,9)$.

The aim of this study was to investigate the effectiveness of IDegAsp in children and adolescents with T1DM with poor glycemic control and acute complications due to noncompliance with insulin injections over a one year period.

\section{Subjects, Methods}

\section{Study Population}

Children and adolescents with T1DM patients with poor glycemic control [HbA1c $>8.5 \% \quad(69.4 \mathrm{mmol} / \mathrm{mol})]$ on basal-bolus regimen (4-5 injections per day) and frequent omission of insulin injections who were switched to IDegAsp (3 injections per day) were included in this study. Inclusion criteria were: diabetes duration of $>1$ year; poor glycemic control on basal bolus regimen; and poor treatment compliance.

Written informed consent was obtained from the parent/ legal guardian of each patient following a detailed explanation of the objectives and protocol of the study, which was conducted in accordance with the ethical principles stated in the "Declaration of Helsinki" and approved by Marmara University Faculty of Medicine Clinical Research Ethics Committee (protocol no: 70737436-050.06.04, date: 07.02.2014).

\section{Assessments}

Patient demographics, body weight and height measurements and pubertal status were retrieved from the hospital records. Data on HbA1c levels, daily insulin doses, the number of basal and bolus insulin injections per day, the number of total severe hypoglycemic episodes per year, self-reported mild to moderate hypoglycemic episodes per week and the number of DKA episodes requiring hospital care were recorded one year before and after the change of the insulin regimen to IDegAsp (Figure 1).

Severe hypoglycemia was defined as an episode requiring the assistance of another person to actively administer carbohydrate, glucagon, or take other corrective action and neurological recovery following the normalization of plasma glucose levels, or both.

\section{Switching to IDegAsp}

Since, the bolus IAsp dose is fixed in IDegAsp (30\%), it was necessary to first determine the main meal which the patient consumed from the dietary history and the relatively fixed and high amount of carbohydrate and the IDegAsp 
injection was tailored to that meal. The dose of IDegAsp was calculated based on the IAsp requirement at that meal which was $30 \%$ of the IDegAsp total dose. In the remaining two main meals, the patients received their usual IAsp dose according to insulin carbohydrate ratios. Further dose adjustments of IDegAsp were made based on postprandial glucose after the IDegAsp meal and fasting glucose levels.

\section{Statistical Analysis}

Statistical analysis was performed using Statistical Package for the Social Sciences Statistics for Windows, version 22.0 (IBM Corp., Armonk, NY, USA). The paired t-test and Mann-Whitney $U$ test were used for analysis of parametric and nonparametric variables, respectively. Data were expressed as mean \pm standard deviation (SD), median minimum-maximum (min-max) and percent (\%) where appropriate. A $p<0.05$ was considered statistically significant.

\section{Results}

\section{Baseline Characteristics}

The study population comprised 50 patients with T1DM, of whom 39 were pubertal and 11 were prepubertal. The mean \pm SD (range) patient age was $12.9 \pm 3.4$ (4-18) years and $28 / 50(56.0 \%)$ were boys. The mean \pm SD (range) duration of diabetes was $5.2 \pm 3.1$ (1.0-13.7) years. Overall, 34 patients were switched to IDegAsp from glargine and 16 patients from detemir as basal insulins. Twenty-three patients were on two doses of basal (total of five injections/ per day) injections with glargine $(n=12)$ or detemir $(n=11)$ and 27 patients were on single basal (total of four injections/ per day) injections with glargine $(n=22)$ or detemir $(n=5)$.

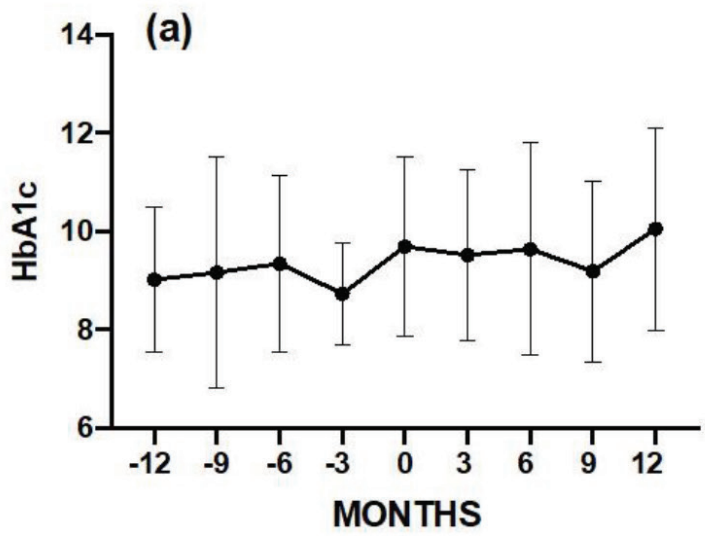

In addition to poor glycemic control and omission of insulin injections, there were frequent episodes of hypoglycemia $(n=11 ; 22 \%)$, excessive daily glucose variability $(n=10 ; 20 \%)$ and frequent DKA $(n=9 ; 18 \%)$ among the subjects.

Twelve $(24 \%)$ patients who were switched to IDegAsp did not want to continue on IDegAsp and switched back to their old regimens. The reasons for discontinuation were persistent hyperglycemia ( $n=7 ; 58.3 \%$ ), difficulty in making dose adjustment due to fixed dose of IAsp within the IDegAsp $(n=4 ; 33 \%)$ and transition to insulin pump therapy $(n=1 ; 8.3 \%)$.

\section{Overall One Year Treatment Outcome with IDegAsp in Those Who Continued}

Overall, 38 (76\%) patients ( 25 boys and 13 girls) completed one year of IDegAsp therapy.

Mean \pm SD and median (min-max) age of this group was $12.8 \pm 3.3$ years and $13.2(4.1-17.7)$ years, respectively.

When values before and after one year of IDegAsp therapy were compared, no significant difference was found in mean body mass index-SD score (BMI-SDS) $(0.34 \pm 1.01 \mathrm{vs}$. $0.21 \pm 1.07, \mathrm{p}=0.26)$ or in mean HbA1c levels $(9.3 \pm 1.7 \%$ vs. $9.6 \pm 1.9 \%, p>0.05$ ) (Table 1). However, one year of IDegAsp therapy was associated with a significant reduction in the number of insulin injections $(4.78 \pm 0.41 \mathrm{vs}$. $3.08 \pm 0.30, p<0.05$ ), total daily insulin dose (basal + bolus insulin: $1.19 \pm 0.34$ vs. $1.01 \pm 0.22 \mathrm{U} / \mathrm{kg} /$ day, $p<0.05)$, long acting/total insulin ratio $(0.46 \pm 0.09$ vs. $0.43 \pm 0.05$, $\mathrm{p}<0.05)$, long acting/rapid acting insulin ratio $(0.91 \pm 0.31$ vs. $0.76 \pm 0.17, p<0.05)$ and long acting insulin dose $(28.75 \pm 13.73$ vs. $23.2 \pm 9.09$ U/day and $0.55 \pm 0.17$ vs.

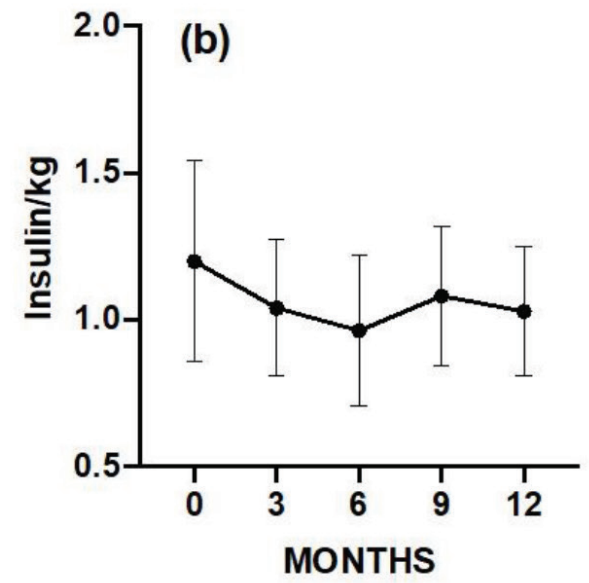

Figure 1. HbA1c levels before (previous year) and after the first year of IDegAsp (a) and insulin dose changes under IDegAsp treatment (b)

HbA1c: hemoglobin A1c, IDegAsp: insulin degludec/insulin aspart 
$0.44 \pm 0.11 \mathrm{U} / \mathrm{kg} / \mathrm{day}, \mathrm{p}<0.05)$ when compared to baseline, pre-switch values (Table 1).

After one year of IDegAsp therapy, a significant decrease was noted in the number of blood glucose-confirmed mild-tomoderate hypoglycemic episodes $(2.1 \pm 1.9$ vs. $0.98 \pm 1.16$ episodes/week, $p<0.05$ ) but not in the total number of severe hypoglycemic episodes (two episodes in two patients before and after therapy, $0.05 \pm 0.22$ episodes per year in each, $p>0.05$ ) when compared to baseline values (Table 1).

Albeit not reaching significance, there was a tendency for a decrease in total DKA episodes/year after one year of IDegAsp therapy when compared to baseline values $(0.28 \pm 0.56$ vs. $0.10 \pm 0.31$ episodes per year, $p>0.05)$. Overall 11 DKA episodes occurred in 9 patients in the year before switching to IDegAsp therapy and 4 DKA episodes were noted in 4 patients after 1 -year of IDegAsp therapy (Table 1).

\section{Insulin Doses at the Third Month of IDegAsp Therapy in Pre- switch Daily Basal Injection Subgroups}

Before switching to IDegAsp, 20 patients were on oncedaily (OD) and 18 patients were on twice-daily (TD) basal injection. There was a non-significant tendency for higher total daily basal and bolus insulin doses in the twice daily vs. OD group at the time of switch.

At the third month of IDegAsp therapy, total $(1.13 \pm 0.27 \mathrm{vs}$. $1.03 \pm 0.19 \mathrm{U} / \mathrm{kg} /$ day in pre-switch OD group and $1.26 \pm 0.41$ vs. $1.07 \pm 0.27 \mathrm{U} / \mathrm{kg} /$ day in pre-switch TD group, $\mathrm{p}<0.05$ for each) and basal $(0.49 \pm 0.15$ vs. $0.44 \pm 0.09 \mathrm{U} / \mathrm{kg} /$ day in OD group and $0.57 \pm 0.21$ vs. $0.47 \pm 0.14 \mathrm{U} / \mathrm{kg} /$ day in TD group, $\mathrm{p}<0.05$ for each) daily insulin doses decreased significantly from baseline, similarly in both the pre-switch OD and preswitch TD subgroups (Table 2).

No significant difference was noted between pre-switch OD and pre-switch TD subgroups in terms of bolus insulin dose (U/kg/day) at baseline vs. the third month of IDegAsp therapy as well as in IDegAsp doses (U/kg/day) at onset and the third month of therapy (Table 2).

\section{Discussion}

IDegAsp is the fixed-ratio co-formulation of two different insulin analogues, which provides an option for longlasting basal insulin coverage and rapid acting post-prandial control in a single injection. It may provide an opportunity to decrease the number of insulin injections and could therefore be preferable in noncompliant patients frequently missing injections. IDegAsp has been confirmed to be non-inferior to IDet + IAsp in terms of HbA1c reduction, together with similar hypoglycemia rates in children (10). Representing the first real-life study evaluating the efficacy of IDegAsp in noncompliant patients, our findings have shown that IDegAsp is non-inferior to basal bolus regimens regarding glycemic control despite fewer injections. Additionally, IDegAsp therapy was associated with lesser likelihood of non-severe hypoglycemia and DKA episodes in the current study. Albeit not statistically significant, a tendency for a lower frequency of DKA episodes was noted after switching to IDegAsp, from 11 DKA episodes in nine patients at baseline to 4 DKA episodes in four patients after the first year of IDegAsp therapy. These data demonstrate the potential of IDegAsp to reduce the rate of metabolic decompensation and DKA in children with T1DM who are

\begin{tabular}{|c|c|c|c|c|}
\hline \multirow[b]{2}{*}{ Mean \pm SD } & & \multicolumn{3}{|c|}{ IDegAsp continuers $(n=38)$} \\
\hline & & Baseline (pre-switch) & One year after IDegAsp & $\mathrm{p}$ value \\
\hline \multicolumn{2}{|l|}{ BMI-SDS } & $0.34 \pm 1.01$ & $0.21 \pm 1.07$ & 0.26 \\
\hline \multicolumn{2}{|c|}{ Number of insulin injections } & $4.78 \pm 0.41$ & $3.08 \pm 0.30$ & 0.001 \\
\hline \multicolumn{2}{|c|}{ Long acting/rapid acting insulin ratio } & $0.91 \pm 0.31$ & $0.76 \pm 0.17$ & 0.009 \\
\hline \multirow[t]{2}{*}{ Rapid acting insulin dose } & U/day & $32.83 \pm 14.63$ & $31.79 \pm 13.3$ & 0.56 \\
\hline & U/kg/day & $0.65 \pm 0.25$ & $0.60 \pm 0.17$ & 0.19 \\
\hline Long acting insulin dose & U/day & $28.75 \pm 13.73$ & $23.2 \pm 9.09$ & 0.0015 \\
\hline
\end{tabular}

"Mean HbA1c levels of the previous year before changing to IDegAsp and first year of IDegAsp treatment.

BMI-SDS: body mass index-standard deviation (SD) score, DKA: diabetic ketoacidosis, IDegAsp: insulin degludec/insulin aspart 
Table 2. Insulin doses at baseline vs. the third month of IDegAsp therapy in pre-switch once-daily and twice daily basal injection subgroups

\begin{tabular}{|c|c|c|c|}
\hline & Baseline (pre-switch) & Third month after IDegAsp & $\mathrm{p}$ value \\
\hline \multicolumn{4}{|l|}{ Total daily doses (Unit/kg/day) } \\
\hline Once-daily basal injection ${ }^{a}(n=20)$ & $1.13 \pm 0.27$ & $1.03 \pm 0.19$ & 0.028 \\
\hline Twice daily basal injection $^{b}(n=18)$ & $1.26 \pm 0.41$ & $1.07 \pm 0.27$ & 0.012 \\
\hline \multicolumn{4}{|l|}{ Basal insulin dose (Unit/kg/day) } \\
\hline Once-daily basal injection ${ }^{\mathrm{a}}(\mathrm{n}=20)$ & $0.49 \pm 0.15$ & $0.44 \pm 0.09$ & 0.046 \\
\hline Twice daily basal injection $^{b}(n=18)$ & $0.57 \pm 0.21$ & $0.47 \pm 0.14$ & 0.037 \\
\hline \multicolumn{4}{|l|}{ Bolus insulin dose (Unit/kg/day) } \\
\hline Once-daily basal injection ${ }^{a}(n=20)$ & $0.62 \pm 0.19$ & $0.60 \pm 0.14$ & 0.66 \\
\hline Twice daily basal injection $^{b}(n=18)$ & $0.70 \pm 0.3$ & $0.68 \pm 0.18$ & 0.78 \\
\hline \multicolumn{4}{|l|}{ IDegAsp dose (Unit/kg/day) } \\
\hline Once-daily basal injection ${ }^{a}(n=20)$ & $0.58 \pm 0.13^{c}$ & $0.63 \pm 0.13$ & 0.34 \\
\hline Twice daily basal injection $^{b}(n=18)$ & $0.61 \pm 0.19^{c}$ & $0.65 \pm 0.16$ & 0.18 \\
\hline
\end{tabular}

${ }^{a}$ Total 4 injections/day, ${ }^{b}$ total 5 injections/day, ${ }^{c}$ calculated IDegAsp dose at the initiation of treatment.

IDegAsp: insulin degludec/insulin aspart

not compliant with insulin injections on a regular basal-bolus regime. This effect seems to be related to the longer duration of action of IDeg, which provides better and durable basal insulin coverage and prevents ketone production unless the patient omits the IDegAsp injection. Similarly, Thalange et al (11) also reported that in children with T1DM, IDegAsp as compared with basal bolus regimen with detemir was associated with a decrease in the rate of ketosis. IDegAsp permits a reduction in the number of injections, which could be additional motivation for the patients and might increase compliance.

Studies of the effectiveness of IDegAsp in children and adolescents with T1DM are scarce $(10,11,12)$. In adult patients with T1DM, OD treatment with IDegAsp and IAsp as bolus insulin for the remaining meals was reported to be associated with significantly lower risk of nocturnal hypoglycemia, improved glycemic control and showed noninferiority compared with IDet + IAsp (13). Although we could not evaluate nocturnal hypoglycemia, real-life experience with IDegAsp in our noncompliant T1 DM patients resulted in a decrease in the total number of non-severe hypoglycemic episodes. The achievement of intensive insulin therapy goals with three injections under IDegAsp + IAsp instead of a minimum of four injections under a conventional basalbolus insulin regimen was considered to be the actual value of this therapy, enabling a reduced injection burden and thereby potentially improving patient adherence and quality of life. Additionally, no significant change in BMI-SDS was observed after switching to IDegAsp in our study, nor in previous studies $(12,14,15,16)$.

Only a few studies in children with IDegAsp have been published and there are no standard protocols for switching doses in children. The switching protocol used in the current study was based on the decreased basal insulin doses with optimal IAsp dose for injection at the meal when IDegAsp was injected to avoid postprandial hypoglycemia. At the end of the third month after initial dose adjustment our patients' insulin doses decreased $11 \%$ in total and $16 \%$ in basal dose. In a pediatric study, dose reduction of $15 \%$ in total daily insulin and $26 \%$ in basal insulin was also reported (10). Thus, when deciding the initial dose of IDegAsp, a 20-30\% reduction in basal insulin dose may be a reasonable and safe starting dose.

In addition, at the end of the third month after initial dose adjustment, insulin doses decreased by $9 \%$ for patients who were on OD basal insulin and by $15 \%$ for those on twice daily basal insulin prior to switching. IDegAsp doses became $0.63 \pm 0.13$ and $0.65 \pm 0.16 \mathrm{U} / \mathrm{kg} /$ day, respectively. Thus, when deciding the initial dose of IDegAsp, previous basal daily injection and doses should be taking into account, together with IAsp dose. Nonetheless, starting with 0.5-0.6 $\mathrm{U} / \mathrm{kg} /$ day or half of the daily total insulin doses seems to be a good strategy.

Although simplified basal-bolus regimen with IDegAsp in T1DM is an attractive option for some patients, a significant number of the patients could not adapt to the treatment and returned to their old regimens. The main reasons for discontinuing IDegAsp therapy were difficulty of dose adjustment and inflexibility of fixed IAsp dose within IDegAsp, which requires a fixed amount of carbohydrate consumption in the meal when IDegAsp is injected. For these reasons, IDegAsp may not be suitable for every patient with T1DM and regimen change should be attempted only after careful evaluation and after a full 
explanation, including advantages and disadvantages, is given to each patient.

\section{Study Limitations}

Although, our study did not have a non-IDegAsp treated control group, treatment with a different regimen in the previous year was used to compare the effectiveness of IDegAsp treatment in the same group of patients. Another limitation of the study was that glucose monitoring and frequency of hypoglycemic episodes were obtained from self-determined blood sugar measurements and self reported by patients/families. Utilizing a continuous glucose monitoring system could have provided a more accurate picture of glycemic control.

\section{Conclusion}

In conclusion, this real-life experience study indicated that IDegAsp was non-inferior to basal-bolus regimen in terms of glycemic control, which was accompanied by a significant reduction in the number of daily injections and frequency of mild-to-moderate hypoglycemic episodes, along with likelihood of lower risk of DKA, in children with T1DM and selected for having poor therapy compliance. Accordingly, use of a simplified basal-bolus regimen with IDegAsp could be an alternative in T1DM patients with frequent hypoglycemia and recurrent DKA, who have poor compliance when having 4-5 injections per day and in whom insulin pump treatment is not available.

\section{Ethics}

Ethics Committee Approval: The study were approved by the Marmara University Faculty of Medicine Clinical Research Ethics Committee (protocol number: 70737436050.06.04, date: 07.02.2014).

Informed Consent: Consent form was filled out by all participants.

Peer-review: Externally peer-reviewed.

\section{Authorship Contributions}

Medical Practices: Tarık Kırkgöz, Mehmet Eltan, Sare Betül Kaygusuz, Zehra Yavaș Abalı, Didem Helvacıoğlu, Tuba Seven Menevşe, Büşra Gürpınar Tosun, Concept: Serap Turan, Design: Tarık Kırkgöz, Tülay Güran, Abdullah Bereket, Serap Turan, Data Collection or Processing: Tarık Kırkgöz, Mehmet Eltan, Sare Betül Kaygusuz, Zehra Yavaş Aball, Didem Helvacıoğlu, Tuba Seven Menevşe, Büşra Gürpınar Tosun, Tülay Güran, Abdullah Bereket, Serap Turan, Analysis or Interpretation: Tarık Kırkgöz, Tülay Güran, Abdullah
Bereket, Serap Turan, Literature Search: Tarık Kırkgöz, Serap Turan, Writing: Tarık Kırkgöz, Abdullah Bereket, Serap Turan.

Financial Disclosure: The authors declared that this study received no financial support.

\section{References}

1. Li R, Zhang P, Barker LE, Chowdhury FM, Zhang X. Cost-effectiveness of interventions to prevent and control diabetes mellitus: a systematic review. Diabetes Care 2010;33:1872-1894.

2. American Diabetes Association. Children and adolescents. Diabetes Care 2015;38(Suppl):70-76

3. Foster C, Bellando J, Wang YC. Diabetes Control and Adherence in Adolescence. Pediatr Ann 2016;45:327-331

4. Heise T, Hövelmann U, Nosek L, Hermanski L, Bøttcher SG, Haahr H. Comparison of the pharmacokinetic and pharmacodynamic profiles of insulin degludec and insulin glargine. Expert Opin Drug Metab Toxicol 2015;11:1193-1201. Epub 2015 Jun 18

5. Jonassen I, Havelund S, Hoeg-Jensen T, Steensgaard DB, Wahlund PO, Ribel U. Design of the novel protraction mechanism of insulin degludec, an ultra-long-acting basal insulin. Pharm Res 2012;29:21042114. Epub 2012 Apr 7

6. Heise T, Nosek L, Roepstorff C, Chenji S, Klein O, Haahr H. Distinct Prandial and Basal Glucose-Lowering Effects of Insulin Degludec/ Insulin Aspart (IDegAsp) at Steady State in Subjects with Type 1 Diabetes Mellitus. Diabetes Ther 2014;5:255-265. Epub 2014 Jun 3

7. Frier BM. Hypoglycaemia in diabetes mellitus: epidemiology and clinical implications. Nat Rev Endocrinol 2014;10:711-722. Epub 2014 Oct 7

8. European Medicines Agency. Ryzodeg ${ }^{\circledR}$. Last Accessed Date: December 2021. Available from: https://www.ema.europa.eu/en/medicines/ human/EPAR/ryzodeg

9. U.S. Food and Drug Administration. Ryzodeg ${ }^{\circledR} 70 / 30$ (insulin degludec and insulin aspart injection). Last Accessed Date: December 2021. Available from: https://www.accessdata.fda.gov/drugsatfda_docs/ label/2016/203313s002lbl.pdf

10. Battelino T, Deeb LC, Ekelund M, Kinduryte O, Klingensmith GJ, Kocova M, Kovarenko M, Shehadeh N. Efficacy and safety of a fixed combination of insulin degludec/insulin aspart in children and adolescents with type 1 diabetes: A randomized trial. Pediatr Diabetes 2018;19:1263-1270. Epub 2018 Aug 16

11. Thalange N, Deeb L, Klingensmith G, Franco DR, Bardtrum L, Tutkunkardas D, Danne T. The rate of hyperglycemia and ketosis with insulin degludec-based treatment compared with insulin detemir in pediatric patients with type 1 diabetes: An analysis of data from two randomized trials. Pediatr Diabetes 2019;20:314-320. Epub 2019 Feb 10

12. Thalange N, Deeb L, Iotova V, Kawamura T, Klingensmith G, Philotheou A, Silverstein J, Tumini S, Ocampo Francisco AM, Kinduryte O, Danne T. Insulin degludec in combination with bolus insulin aspart is safe and effective in children and adolescents with type 1 diabetes. Pediatr Diabetes 2015;16:164-176. Epub 2015 Feb 12

13. Hirsch IB, Franek E, Mersebach H, Bardtrum L, Hermansen K. Safety and efficacy of insulin degludec/insulin aspart with bolus mealtime insulin aspart compared with standard basal-bolus treatment in people with Type 1 diabetes: 1-year results from a randomized clinical trial (BOOST ${ }^{\circledR}$ T1). Diabet Med 2017;34:167-173. Epub 2016 Feb 1

14. Onishi Y, Ono Y, Rabøl R, Endahl L, Nakamura S. Superior glycaemic control with once-daily insulin degludec/insulin aspart versus insulin 
glargine in Japanese adults with type 2 diabetes inadequately controlled with oral drugs: a randomized, controlled phase 3 trial. Diabetes Obes Metab 2013;15:826-832. Epub 2013 Apr 5

15. Kaneko S, Chow F, Choi DS, Taneda S, Hirao K, Park Y, Andersen TH, Gall MA, Christiansen JS; BOOST: Intensify All Trial Investigators. Insulin degludec/insulin aspart versus biphasic insulin aspart 30 in Asian patients with type 2 diabetes inadequately controlled on basal or pre-/self-mixed insulin: a 26-week, randomised, treat-to-target trial. Diabetes Res Clin Pract 2015;107:139-147. Epub 2014 Oct 14
16. Dardano A, Bianchi C, Del Prato S, Miccoli R. Insulin degludec/insulin aspart combination for the treatment of type 1 and type 2 diabetes. Vasc Health Risk Manag 2014;10:465-475. 